\title{
Craving por crack: uma revisão da literatura
}

\section{Craving by crack users: a literature review}

\section{Craving de crack: una revisión de la literatura}

\author{
Daniel Galeno Machado ${ }^{1}$, Fernando José Guedes da Silva Júnior ${ }^{2}$, \\ Larissa Alves de Araújo Lima ${ }^{3}$, Claudete Ferreira de Souza Monteiro ${ }^{4}$ \\ ${ }^{1}$ Enfermeiro. Mestrando em Enfermagem pela Universidade Federal do Piauí (UFPI). Bolsista Capes. \\ ${ }^{2}$ Enfermeiro. Mestrando em Enfermagem pela Universidade Federal do Piauí (UFPI). Bolsista Capes. \\ ${ }^{3}$ Acadêmica de Enfermagem da Universidade Federal do Piauí (UFPI). Bolsista Pibic/CNPq. \\ ${ }^{4}$ Enfermeira. Doutora em Enfermagem. Professora do Programa de Pós-Graduação Mestrado em Enfermagem da Universidade \\ Federal do Piauí (UFPI). \\ Cómo citar este relato en edición digital: Galeno Machado, D., Guedes da Silva Junior, F.J., Alves de Araújo Lima, L., Ferreira de \\ Souza Monteiro, C. (2014) Craving por crack: uma revisão da literatura. Cultura de los Cuidados (Edición digital) 18, 38. Disponi- \\ ble en: http://dx.doi.org/10.7184/cuid.2014.38.14>
}

Correo electónico: danielgalenoo@hotmail.com Recibido: 12/10/2013; Aceptado: 04/01/2014

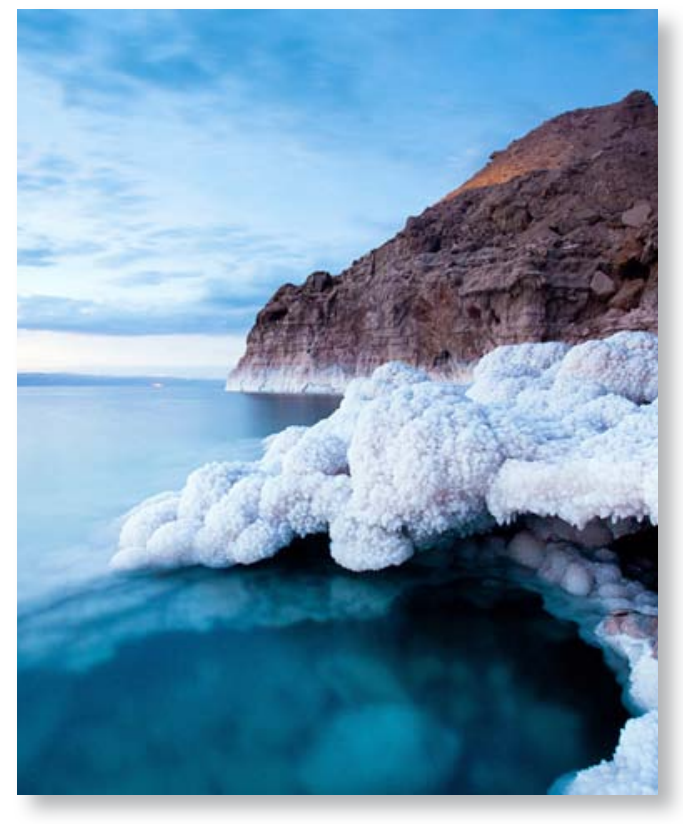

\section{ABSTRACT}

This study aimed to raise the scientific literature on the constructs of craving relating it to situations that stimulate the usage pattern of the crack, the repercussions on the lives of users and control strategies. We conducted a review of the literature through databases SciELO, LILACS and MEDLINE, using the keywords: craving, crack cocaine, and drug dependence. The survey period was from 1996 to 2012, totaling 39 studies. The results demonstrated that the concept of craving (or tear) most used is understood as a strong desire to use a substance, which may influence the user's life in a negative way through relational ruptures and illicit activities. The craving management is through various strategies and techniques ranging from the use of other drugs to drug therapy. It is concluded that the craving because of its multifactorial etiology and on the way management becomes his peculiar understanding to help crack users in a unique way, involving social and psychological peculiarities of each.

Keywords: Crack Cocaine, Craving, Chemical Dependency

\section{RESUMEN}

Este estudio tuvo como objetivo revisar la literatura científica sobre las construcciones de deseo en relación a las situaciones que estimulan el patrón de uso del crack, las repercusiones en la vida de los usuarios y las estrategias de control. Se realizó una revisión de la literatura 
a través de bases de datos SciELO, LILACS y MEDLINE, usando las palabras clave: Deseo, el crack y la dependencia de drogas. El período de estudio fue de 1996 a 2012, un total de 39 estudios. Los resultados demostraron que el concepto de deseo (o desgarro) más utilizado se entiende como un fuerte deseo de utilizar una sustancia, que puede influir en la vida del usuario de una manera negativa a través de rupturas relacionales y actividades ilícitas. La gestión deseo es a través de diversas estrategias y técnicas que van desde el uso de otros fármacos para la terapia con medicamentos. Se concluye que el ansia por su etiología multifactorial y sobre la forma de gestión se convierte en su peculiar entendimiento para ayudar a los consumidores de crack de una manera única, con la participación peculiaridades sociales y psicológicas de cada uno.

Palabras clave: Cocaína Crack, Deseo, Dependencia Química

\section{RESUMO}

Este estudo objetivou levantar na literatura científica os constructos acerca do craving relacionando-o às situações que estimulam o padrão de uso do crack, as repercussões na vida dos usuários e as estratégias de controle. Realizou-se uma revisão narrativa da literatura por meio das bases de dados SciELO, LILACS e MEDLINE, utilizando os descritores: craving, cocaíne crack, dependence e drug. O período pesquisado foi de 1996 a 2012, totalizando 39 estudos. Os resultados demonstraram que o conceito do craving (ou fissura) mais utilizado é entendido como um forte desejo de utilizar determinada substância, o qual pode repercutir na vida do usuário de forma negativa por meio de rupturas relacionais e atividades ilícitas. O manejo do craving se dá por meio de várias estratégias e técnicas que incluem desde o uso de outras drogas até a terapia medicamentosa. Conclui-se que o craving por conta de sua multifatorialidade quanto à etiologia $\mathrm{e}$ forma de manejo torna-se peculiar o seu entendimento para ajudar os usuários de crack de modo singular, envolvendo peculiaridades sociais e psicológicas de cada um.

Palavras-chave: Cocaína Crack, Craving, Dependência Química

\section{INTRODUÇÃO}

Profissionais da área da saúde voltam seu olhar em busca de compreenderem fatores que estimulam o consumo de crack em adolescentes e jovens em todo o mundo. O crack torna-se uma das drogas mais procuradas, consumidas e de dificil tratamento. Considerar o desejo, incontrolável, de consumir crack, é hoje um dos fatores que ainda necessita de conhecimento que desejo é este? Como se chama? Que estratégias já despontam para diminuir esta vontade? Questões como estas são abordadas neste estudo mostrada por meio das experiencias de alguns profissionais e já apontadas como caminhos no tratamento do crack.

Este desejo, cada vez mais crescente pelo crack e outras drogas, entre seus usuários é conhecido em algumas literatura por craving e ainda não traz uma definição consensual fazendo com que os pesquisadores da área de dependência química não se limitem a uma só designação (Araújo et al., 2008). Entretanto, àquela mais comumente utilizada é a de que o craving é um impetuoso desejo de utilizar determinada substância (Kozlowski et al., 1996).

Outros pesquisadores, também, definiram o craving como um pensamento obsessivo, um forte desejo de experienciar os efeitos adquiridos após o uso de uma substância específica, 
um insuperável impulso para utilizar a droga, uma necessidade para saciar os sintomas de abstinência, uma esperança de resultado positivo e um incentivo para utilizar a substância (Araújo et al., 2008; Singleton; Anderson; Heishman, 2003).

O craving, também conhecido como "fissura" - termo popularmente utilizado pelo usuário de drogas no Brasil - é uma variável importante a ser analisada, sobretudo, no tratamento da dependência do crack devido a vulnerabilidade do usuário exposto à momentos de recaída e/ou abandono da terapêutica utilizada (Chaves et al., 2011).

Na dependência do crack, o craving torna-se ainda mais peculiar por conta da descrição dada pelos usuários de incontrolável e com uso repetitivo e compulsivo levando-os à um padrão de consumo torrente em vários dias subsequentes até o alcance do seu próprio esgotamento biopsicossocial (Ribeiro; Sanchez; Nappo, 2010). Este tipo de consumo descomedido e contínuo é denominado de binge e pode permanecer até que o suprimento de crack cesse ou que haja exaustão do usuário (Nappo; Sanchez; Oliveira, 2011).

Diante dessa problemática e da importância da compreensão do craving como fator que irá contribuir para adesão ao tratamento do consumo de crack entre seus usuários, este estudo objetivou levantar na literatura científica os constructos deste fenômeno relacionando-o às situações que estimulam o padrão de uso, as repercussões na vida dos usuários e as estratégias de controle apontadas.

\section{MÉTODO}

Trata-se de uma revisão da narrativa e compreensiva de estudos e pesquisas sobre craving. Considerou-se a busca recente da força da evidencia em estudos com diferentes abordagens, haja vista que o fenômeno pesquisado parte tanto da objetividade quando da subjetividade do sujeito.

Foram acessados as bases de dados SciELO, LILACS e MEDLINE, por meio dos descritores: "craving", "cocaíne crack", "dependence", "drug". O estudo abrangeu publicações a partir dos anos de 1996 a 2012, sendo incluidas aqueles que atendiam ao tema e discorriam sobre o craving, totalizando 39 estudos.

Os eixos temáticos principais apresentados se referiam a conceitos, estímulos, repercussões e estratégias sobre o craving por crack. Assim, buscou-se destacar a força destes eixos sistematizadados em três categorias: bases conceituais, repercussões sociais e estratégias para manejo do crack.

\section{RESULTADOS E DISCUSSÃO \\ Craving e suas bases conceituais}

O conceito mais utilizado de craving atribui ser um forte desejo para consumir uma substância específica (Kozlowski et al., 1996). Entretanto, Sayette et al. (2000) o definem de forma mais ampla, abrangendo não somente o desejo, mas também como a intenção de utilizar a droga para satisfazer este desejo, antecipando desse modo os efeitos positivos associados ao seu uso e a diminuição do afeto negativo e dos sintomas relacionados à abstenção.

Porém, Kozlowski et al. (1996) afirmam que a intenção de utilizar a droga para satisfazer o desejo intenso, as expectativas de resultado quanto aos seus efeitos e o craving pela droga não são termos que podem ser definidos em uma mesma classificação, pois são conceitualmente diferentes, sendo, a intenção e as expectativas consequências posteriores do próprio craving. 
O craving pode ocorrer tanto na fase de consumo quanto no início da abstinência da droga, ou mesmo após um longo tempo sem sua utilização, que pode ser desencadeado através de momentos, imagens, sons e odores - estímulos externos - ou emoções e estados de humor - estímulos internos - que remetam às situações de uso; isto se dá com o uso repetido, fazendo com que esses estímulos condicionados ao uso da droga passem a construir uma teia associativa acumulada na memória (Araújo et al., 2004; Zeni; Araújo, 2011).

Ambos os estímulos - externos ou internos -, também são conhecidos como gatilhos que, poderão ativar o craving, quando o usuário de crack é exposto aos mesmos, desencadeando, assim, um desejo intenso de procurar à droga e seu consumo (Conklin; Tiffany, 2002). Zeni e Araújo (2009) ressaltam que a ativação por esses estímulos dependem do histórico de uso individual do crack de cada paciente, como demonstra sua pesquisa, na qual os usuários afirmaram despertar esses gatilhos quando sentiram algum tipo de emoção (raiva tristeza, alegria), ou por estímulos provenientes do ambiente como uso do álcool, dinheiro em mãos, ou mesmo em ver usuários de crack.

Zeni e Araújo (2011) expuseram aos usuários de crack oito imagens fotográficas, durante um minuto, as quais remetessem ao uso do crack - como "pedras", cachimbos e latas - afim de que o craving fosse induzido. Além desta tentativa de indução, os comportamentos e trejeitos peculiares dos próprios usuários, quando foram apresentadas as fotografias, também emergiram de forma a contribuir a ativação dos gatilhos preservados em suas memórias.

Em outro estudo de Zeni e Araújo (2009), usando a mesma exposição de imagens feita anteriormente, observaram que alguns usuá- rios de crack negaram indução do craving, devido ao fato da dificuldade de reconhecimento do craving em si e/ou pelo temor que, ao relatarem esse desejo, tenham suas altas adiadas. Outros também afirmaram que não sentiam esse desejo, pois estavam em ambiente isolado do mundo exterior, consequentemente, afastado da possibilidade de acesso à droga.

Os sintomas de ansiedade, também, devem ser variáveis relatadas na indução do craving, tendo este uma correlação positiva quando os níveis de sintomas ansiosos aumentam, e vice-versa (Almeida; Araújo, 2005). Além de ansiedade, os sintomas depressivos, também configuram como indutores da elevação de craving como apresentam Araújo et al. (2007), indicando a quantidade de cigarros consumida por dia com a gravidade do desejo por tabaco e com sintomas de depressão e ansiedade.

Cavallo e Pinto (2001) e Delfino, Jamner e Whalen (2001) analisaram a intensidade dos sintomas ansiosos e depressivos e o consequente aumento do craving em usuários de nicotina através da privação de dieta e da diferença entre gêneros, respectivamente. Os mesmos achados Castro et al. (2008) encontraram ao analisarem a gravidade do nível de fissura em mulheres tabagistas deprimidas, impulsionadas pela tentativa de aliviar seu afeto negativo.

Field e Duka (2004) observaram que as mulheres tinham um craving mais intenso do que os homens ao serem apresentados aos estímulos relacionados ao tabaco. Quanto ao craving pela Cannabis, não foi encontrada diferença significante entre sua severidade por Cannabis e os sintomas de ansiedade e depressão (Polese et al., 2011). Resultado contrário ao obtido na revisão feita por Andrade e Argimon (2008) que constataram uma maior associação entre sintomas ansiosos e depressivos 
naqueles adolescentes cujo uso de Cannabis era intenso e regular.

Referente ao craving no início da abstinência de crack, Balbinot et al. (2011) conferem que a intensidade do mesmo não está relacionada à quantidade de substância utilizada pelo usuário, mas com o tempo de abstenção da mesma. Isto implica que quanto mais recente for o último uso de crack, maior é a necessidade avaliada.

\section{Repercussões sociais dos efeitos do craving na vida dos usuários de crack}

A obsessão gerada pelo craving por crack desnorteia o indivíduo em suas condutas e fazem com que a capacidade de discernimento e autonomia de seus atos diminuam, focando suas atitudes, tão somente, no desejo de consumir mais crack, apesar do desgaste físico, psíquico e moral (Chaves et al., 2011).

Esse comportamento compulsivo se faz mediante o padrão de consumo intenso, contínuo e repetitivo de crack, denominado binge, que por sua vez, é provocado pelo craving e pode durar dias até que o suprimento de droga finde, ou que haja a exaustão do usuário através do seu mal-estar físico e psicológico (Nappo; Sanchez; Oliveira, 2011).

Uma vez que o crack adquiriu lugar de extrema importância na vida do indivíduo são realizadas por este, atividades que põe em risco sua integridade física e moral. Assim, com os valores rebaixados, o indivíduo amplia as possibilidades de obtenção de crack e/ou dinheiro para comprá-lo, incluindo atividades ilícitas (Chaves et al., 2011).

Dessa forma, a urgência pelo uso do crack por meio desse padrão compulsivo e a falta de condições financeiras para suprir sua demanda expõem o usuário em situação de fragilidade, levando-o à estratégias arriscadas para obtenção de drogas e a violência intrafamiliar (Oliveira; Nappo, 2008).

Em relação à presença de violência, Seleghim et al. (2011) em sua pesquisa realizada com usuários de crack do interior do Paraná, constata a frequência de eventos agressivos como brigas e discussões entre os familiares e o usuário acarretando em atitudes repressivas de encarceramento domiciliar e denúncia à polícia contra o usuário.

Eleghim et al. (2011) ainda evidenciam, além da ruptura familiar, a ruptura conjugal, quando relatos de ex-cônjuges comprovam conflitos relacionais, caracterizados por desentendimentos e divergências, e constantes cobranças para cessar o uso da droga. Desta forma, alguns usuários relataram o fim do relacionamento em decorrência do consumo intenso de crack.

Quanto a obtenção de crack por meio de atividades ilícitas, Guimarães et al. (2008) apontam que grande parte dos seus sujeitos pesquisados possuíam antecedentes criminais alegando o crime por motivação de uso da droga. Isto se dá por conta da necessidade que acarreta a busca incessante pela droga, facilitando assim, a prática de atividades ilegais para a maior obtenção do crack.

Essas são condutas que podem ocasionar homicídios na população de usuários como revela o estudo de Sant'anna, Aerts e Lopes (2005) sobre a situação de vulnerabilidade de morte em adolescentes de Porto Alegre, apontando que dentre as principais causas desses homicídios, destacava-se a vingança, participação em assaltos ou queima de arquivo e envolvimento com drogas.

Outro estudo revela que não somente adolescentes, como também, adultos jovens denotam uma população exposta ao risco de morte por homicídios, devido o uso de crack levar ao 


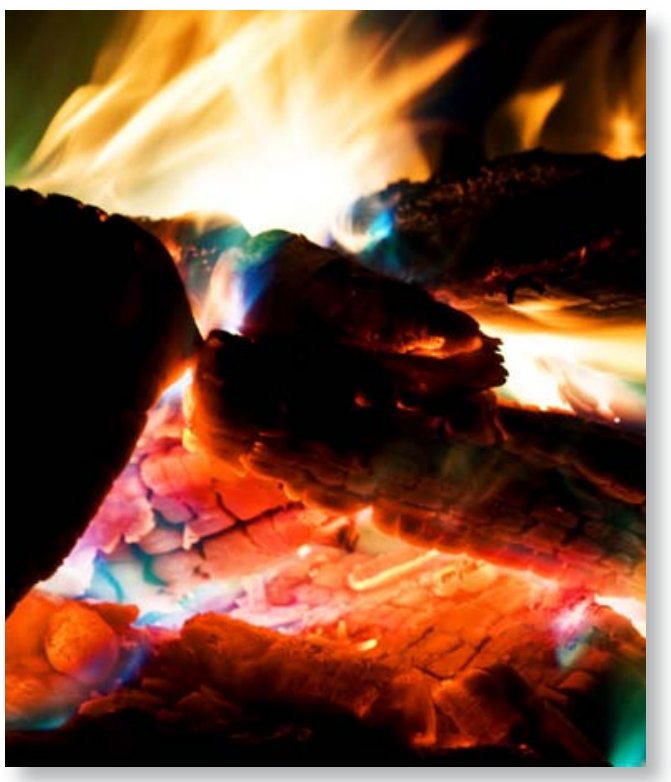

roubo, violência e, inclusive, endividamento com traficantes (Chesnais, 1999).

Cross et al. (2001) estudaram quais os hábitos mais utilizados pelos usuários de crack norte-americanos para manterem o uso contínuo da droga, dentre eles a realização de tráfico do próprio crack, roubos motivados pelos efeitos da droga, sequestros, venda de pertences próprios e de familiares, golpes financeiros de maneiras diversas e prostituição.

Essa última fora relatada por Oliveira e Nappo (2008) como prática para obtenção de cra$\mathrm{ck}$, tanto de mulheres quanto de homens. Neste estudo, foi visto que metade das mulheres praticavam a prostituição para a aquisição de dinheiro e a posterior compra da droga; entretanto, é visto a prostituição compulsória, em que casais de usuários pactuavam um acordo com traficantes, no qual consistia na permissão dos homens para o uso sexual de suas parceiras, fazendo com que os homens consentissem o uso de suas parceiras por outrem.

Além dessa nova modalidade de prostituição, esta atividade tem deixado de ser exclusivamente feminina. Os homens, também, já trocaram sexo por crack com a prática no mesmo sexo, apesar de não se declararem homossexuais (Chaves et al., 2011; Oliveira; Nappo, 2008).

Com estudo executado na população norte-americana Maranda, Han e Rainone (2004) verificaram a prostituição de ambos os sexos, entretanto, com ressalvas no intuito do seu objetivo. Os mesmos sugerem que enquanto a prostituição feminina seria impulsionada somente pela fissura por crack, a masculina se contrapõe, além de ser praticada devido ao forte desejo pela droga, haveria a ocorrência de aumento da libido sexual induzido pelo efeito da droga.

Entretanto, a prática da prostituição pode ser feita de forma negligenciada através do não uso de preservativos, visto que este tipo de proteção é tido de forma secundária, visando apenas o lucro desta atividade (Chaves et al., 2011). Em pesquisa realizada por Schönnesson et al., 2008), houve uma correlação positiva direta com um grupo de usuários de crack que mantém comportamento sexual de risco e altas taxas de execução de meios ilegais para obtenção de dinheiro e maior prevalência de padrão binge de uso de crack.

Além da prostituição, Chaves et al. (2011), também investigaram outras ocorrências na vida do usuário devido ao craving, como a manipulação de pessoas, na qual o usuário lança artifícios como a mentira e dissimulação mudando, muitas vezes, sua personalidade e perdendo a confiança de pessoas significativas e ainda proporcionado a exposição do usuário de crack ao frequentar lugares violentos, como favelas e bocas de fumo.

Estratégias e técnicas no manejo do craving

Ribeiro, Sanchez e Nappo (2010) registraram nove diferentes estratégias elaboradas 
por usuários de crack no controle do craving. Dentre elas, pode-se destacar a associação com outras drogas como maconha e álcool. Os usuários descrevem que a substituição do crack pelo mesclado, contribui diminuindo assim seus efeitos, além de gerar padrão compulsivo menos intenso.

Já o álcool, é mais utilizado para amenizar o medo e a agressividade, decorrentes do craving, além de aliviar o próprio desejo incontrolável. Com essa conduta os usuários relataram cessar a vontade constante de uso do crack em detrimento ao consumo de bebidas alcoólicas, fazendo com que este sirva de "calmante", reduzindo a energia para a busca de dinheiro e/ ou droga, e como consequência, servindo de controle para o intenso desejo (Ribeiro; Sanchez; Nappo, 2010).

Desviar o pensamento do desejo pelo crack é importante para seu enfrentamento. Assim, ocupar-se com outras formas de sentir prazer constitui como uma estratégia eficaz para diminuir o pensamento obsessivo pela droga. Atividades como trabalhar, estudar, frequentar uma religião, praticar esportes, dormir e ter relação sexual são alternativas para diminuir a fissura por crack (Chaves et al., 2011).

Segundo Chaves et al. (2011), alguns usuários quando sentem a necessidade de cessação do uso de crack, empregavam estratégias focadas na mudança de comportamento como medidas de redução de danos. Assim, evitar o contexto social de uso do crack, afastar-se do local e dos amigos de consumo e até procurar uma internação em leitos psiquiátricos configuravam-se como estratégias de controle para a interrupção do craving.

Além disso, pesquisadores na área da dependência química estão desenvolvendo técnicas para a diminuição do craving, logo nos primeiros dias de interrupção de uso da dro- ga. Agentes farmacológicos como a pergolida foram utilizadas em ensaios clínicos abertos com usuários de cocaína concomitante á grupos controlados de placebo. Contudo, o efeito placebo comparado aos efeitos do fármaco mostrou-se igual quando mensurado antes e depois da intervenção farmacológica (Focchi; Leite, 2001).

Reis, Castro e Laranjeira (2008) constataram que mais da metade de sua população pesquisada, também usuários de cocaína, não houve redução da intensidade e duração do craving com a utilização do topiramato. Entretanto, esta mesma droga mostrou-se bastante eficaz no controle do craving por álcool apresentando um fármaco promissor no tratamento da dependência de bebidas alcoólicas (Castro; Baltieri, 2004; Johnson et al., 2003). Já, em relação à nicotina o uso da bupropiona é mais utilizado a fim de diminuir o craving em pacientes abstinentes (Balbani; Montovani, 2005).

Outras técnicas, também, podem ser citadas, ainda que suas bases científicas estejam sendo pesquisadas em populações específicas de usuários de droga, como o uso de alimentos ricos em carboidratos como a glicose e sacarose para aplacar o desejo intenso nos primeiros dias de abstinência de bebidas alcoólicas (Toffolo et al., 2011; Krahn et al., 2006; Cowan; Devine, 2008).

Em usuários de crack, o emprego de modalidades terapêuticas como, jogos cooperativos, desenvolvimento de habilidades para o domínio e adaptação das situações de estresse (denominado de coping) e o trabalho das funções musculoesqueléticas que reforcem as atividades de relaxamento respiratório propicia; a ampliação de técnicas e estratégias para o controle do craving (Alves; Araújo, 2012; Araújo; Oliveira; Cemi, 2011; Araújo et al., 2010). 
Estas inúmeras estratégias e técnicas de manejo do craving vem sendo desenvolvidas na perspectiva de contribuir para redução dos sintomas da abstinência, adesão ao processo terapêutico e, sobretudo, para prevenção da recaída.

\section{CONCLUSÃO}

Por conta da multifatorialidade quanto a sua etiologia, o craving requer novas reflexões embasadas em novas pesquisas a fim de se buscar outras formas de entendimento do seu manejo tanto para lidar com as repercussões na vida dos usuários quanto nas estratégias de controle de uso.

Quanto às estratégias, devem ser peculiares de cada usuário pesquisado e do contexto em que o mesmo se insere. Padronizar estratégias pode não funcionar com todos os usuários. Essas condições são particulares dependendo da experiência que cada um possui sobre seu fenômeno e sua relação com os valores morais e momentos de vida de cada indivíduo.

Mesmo que a utilização de fármacos, o uso de alimentos ricos em carboidratos e outras modalidades de manejo do craving estejam sendo empregadas em pesquisas com populações específicas de usuários de drogas, ainda faz-se necessário novos estudos com delineamentos metodológicos específicos que subsidiem a avaliação das técnicas de controle do craving em usuários de crack.

Ainda assim, valorizar o entendimento da dinâmica de vida do usuário de crack, tais como as questões que os permeiam - violência, situações de risco, eventos criminais e prostituição - tem papel fundamental no entendimento do contexto social que cerca estes usuários com a finalidade de uma assistência integral para essa população e estimular mudança de comportamento e de ideias em busca do consumo de crack.

\section{REFERÊNCIAS}

- Almeida, S.C., Araújo, R.B. (2005) Avaliação da efetividade do relaxamento na variação dos sintomas da ansiedade e da fissura em pacientes em tratamento de alcoolismo. Boletim Saúde. 19(2):135-42.

- Alves, G.S.L., Araújo, R.B. (2012) A utilização dos jogos cooperativos no tratamento de dependentes de crack internados em uma unidade de desintoxicação. Rev Bras Med Esporte. 18(2):77-80.

- Andrade, T.M.R., Argimon, I.I.L. (2008) Sintomas depressivos e uso de cannabis em adolescentes. Rev Psicol Estud. 13(3):567-73.

- Araújo, RB., Oliveira, M.M.A., Cemi, J. (2011) Desenvolvimento de Role-Playing Game para prevenção e tratamento da dependência de drogas na adolescência. Psicologia: Teoria e Pesquisa. 27(3):347-56.

- Araújo, R.B., Oliveira, M.S., Moares, J.F.D., Pedroso, R.S., Port, F., Castro, M.G.T. (2007) Validação da versão brasileira do Questionnaire of Smoking Urges-Brief. Rev Psiq Clín. 34(4):166-75.

- Araújo, R.B., Oliveira, M.S., Nunes, M.L.T., Piccoloto, L.B., Melo, W.V. (2004) A avaliação do craving em alcoolistas na síndrome de abstinência. Psico-USF. 9(1):71-6.

- Araújo, R.B., Oliveira, M.S., Pedroso, R.S., Miguel, A.C., Castro, M.G.T. (2008) Craving e dependência química: conceito, avaliação e tratamento. J Bras Psiquiatr. 57(1):57-63.

- Araújo, R.B., Pansard, M., Boeira, B.U., Rocha, N.S. (2010) As estratégias de coping para o manejo da fissura de dependentes de crack. Rev HCPA. 30(1):36-42.

- Balbani, A.P.S., Montovani, J.C. (2005) Métodos para abandono do tabagismo e tratamento da dependência da nicotina. Rev Bras Otorrinolaringol. 71(6):820-7.

- Balbinot, A.D., Alves, G.S.L., Amaral Junior, A.F., Araújo, R.B. (2011) Associação entre fissura e perfil antropométrico em dependents de crack. J Bras Psiquiatr. 60(3):205-9.

- Castro, LA; Baltieri, DA. (2004) Tratamento farmacológico da dependência do álcool. Rev Bras Psiquiatr., São Paulo, 26(Supl):43-6. 
- Castro, M.G.T., Oliveira, M.S., Araújo, R.B., Pedroso, R.S. (2008) Relação entre gênero e sintomas depressivos e ansiosos em tabagistas. Rev Psiquiatr RS, 30(1):25-30.

- Cavallo, D.A., Pinto, A. (2001) Effects of mood induction on eating behavior and cigarette craving in dietary restrainers. Eat Behav. 2(2):113-27.

- Chaves, T.V., Sanchez, Z.M., Ribeiro, L.A., Nappo, S.A. (2011) Fissura por crack: comportamentos e estratégias de controle de usuários e ex-usuários. Rev Saúde Pública. 45(6):1168-75.

- Chesnais, J.C. (1999) A violência no Brasil: causas e recomendações políticas para a sua prevenção. Ciênc Saude Coletiva. 4(1):53-69.

- Conklin, A.C., Tiffany, S.T. (2002). Applying extinction research and theory to cue-exposure addiction treatments. Addiction. 97:155-67.

- Cowan, J., Devine, C. (2008) Food, eating, and weight concerns of men in recovery from substance addiction. Appetite. 50(1):33-42.

- Cross, J.C. Johnson, B.D., Davis, W.R., Liberty, H.J. (2001) Supporting the habit: income generation activities of frequent crack users compared with frequent users of other hard drugs. Drug Alcohol Depend. 64(2):191-201.

- Delfino, R.J., Jamner, L.D., Whalen, C.K. (2001) Temporal analysis of the relationship of smoking behavior and urges to mood states in men versus women. Nicotine Tob Res. 3(3):235-48.

- Field, M., Duka, T. (2004) Cue reactivity in smokers: the effects of perceived cigarette availability and gender. Pharmacol Biochem Behav. 78(3):647-52.

- Focchi, G.R.A., Leite, M.C. Scivoletto, S. (2001) Utilização do agonista dopaminérgico pergolida no tratamento da "fissura" por cocaína. Rev Bras Psiquiatr. 23(4):188-94.

- Guimarães, C.F., Santos, D.V.V. Freitas, R.C., Araújo, R.B. (2008) Perfil do usuário de crack e fatores relacionados à criminalidade em unidade de internação para desintoxicação no Hospital Psiquiátrico São Pedro de Porto Alegre (RS). Rev Psiquiatr RS, 30(2):101-8.

- Johnson, B.A., Ait-Daoud, N., Bowden, C.L., DiClemente, C.C., Roache, J.D., Lawson, K., Javors, M.A. (2003) Oral topiramate for treatment of alcohol dependence: a randomized controlled trial. The Lancet. 36(1):1677-85.
- Kozlowski, L.T., Pillitteri, J.L., Sweeney, C.T., Whitfield, K.E., Graham, J.W. (1996) Asking questions about urges or cravings for cigarettes. Psychology of Addict Behav. 10(4):248-60.

- Krahn, D., Grossman, J., Henk, H., Mussey, M., Crosby, R., Gosnell, B. (2006) Sweet intake, sweet-liking, urges to eat, and weight change: relationship to alcohol dependence and abstinence. Addict Behav. 3(4):622-31.

- Maranda, M.J., Han, C., Rainone, G.A. (2004) Crack cocaine and sex. J Psychoactive Drugs. 36(3):315-22.

- Nappo, SA; Sanchez, ZVDM; Oliveira, LG. (2011) Crack, AIDS, and women in São Paulo, Brazil. Subst Use Misuse. $46(4): 476-85$.

- Oliveira, L.G., Nappo, S.A. (2008) Caracterização da cultura de crack na cidade de São Paulo: padrão de uso controlado. Rev Saúde Pública. 42(4):664-71.

- Polese, G.P., Zardo, E., Bertão, E.S., Gonçalves, P.C., Pedroso, R.S., Castro, M.G.T., Araújo, R.B. (2011) O craving, sintomas de ansiedade e depressão em usuários de Cannabis. Revista HCPA. 31(3):303-10.

- Reis, A.D., Castro, L.A., Laranjeira, R. (2008) Craving decrease with topiramate in outpatiente treatment for cocaine dependence: na open label trial. Rev Bras Psiquiatr. 30(2):132-5.

- Ribeiro, L.A., Sanchez, Z.M., Nappo, A.S. (2010) Estratégias desenvolvidas por usuários de crack para lidar com os riscos decorrentes do consumo da droga. J Bras Psiquiatr. 59(3):210-8.

- Sant'Anna, A., Aerts, D., Lopes, M.J. (2005) Homicídios entre adolescentes no Sul do Brasil: situação de vulnerabilidades segundo seus familiares. Cad Saude Publica. 21(1):120-9.

- Sayette, MA; Shiffman, S; Tiffany, S; Niaura, R; Martin, CS; Shadel, W. (2000) The measurement of drug craving - methodological aproaches to craving research. Addiction. 95(2):189-210.

- Schonnesson, L.N., Atkinson, J., Williams, M.L., Bowen, A., Ross, M.W., Timpson, S.C. (2008) A cluster analysis of drug use and sexual HIV risks and their correlates in a sample of African-American crack cocaine smokers with HIV infection. Drug Alcohol Depend., Nova York, 97(2):44-53. 


\section{Cultura de las Cuidados}

- Seleghim, M.R., Marangoni, S.R., Marcon, S.S. Oliveira, M.L.F. (2011) Vínculo familiar de usuários de crack atendidos em uma unidade de emergência psiquiátrica. Rev Latino-Am. Enfermagem. 19(5):118-25.

- Singleton, E.G., Anderson, L.M., Heishman, S.J. (2003) Reliability and validity of the tobacco craving questionnaire and validation of craving-induction procedure using multiple measures of craving and mood. Addiction. 98(3):1537-46.
- Toffolo, M.C.F., Pereira, I.S., Silva, K.A.L., Marliére, C.A., Nemer A.S.A. (2011) Escolha de alimentos durante a abstinência alcoólica: influência na fissura e no peso corporal. J Bras Psiquiatr.60(4):341-6.

- Zeni, T.C., Araújo, R.B. (2009) O relaxamento respiratório do manejo do craving e dos sintomas de ansiedade em dependentes de crack. Rev Psiquiatr RS, 1(2):116-9.

- Zeni, T.C; Araújo, RB. (2011) Relação entre o craving por tabaco e o craving por crack em pacientes internados para desintoxicação. J Bras Psiquiatr. 60(1):28-33.

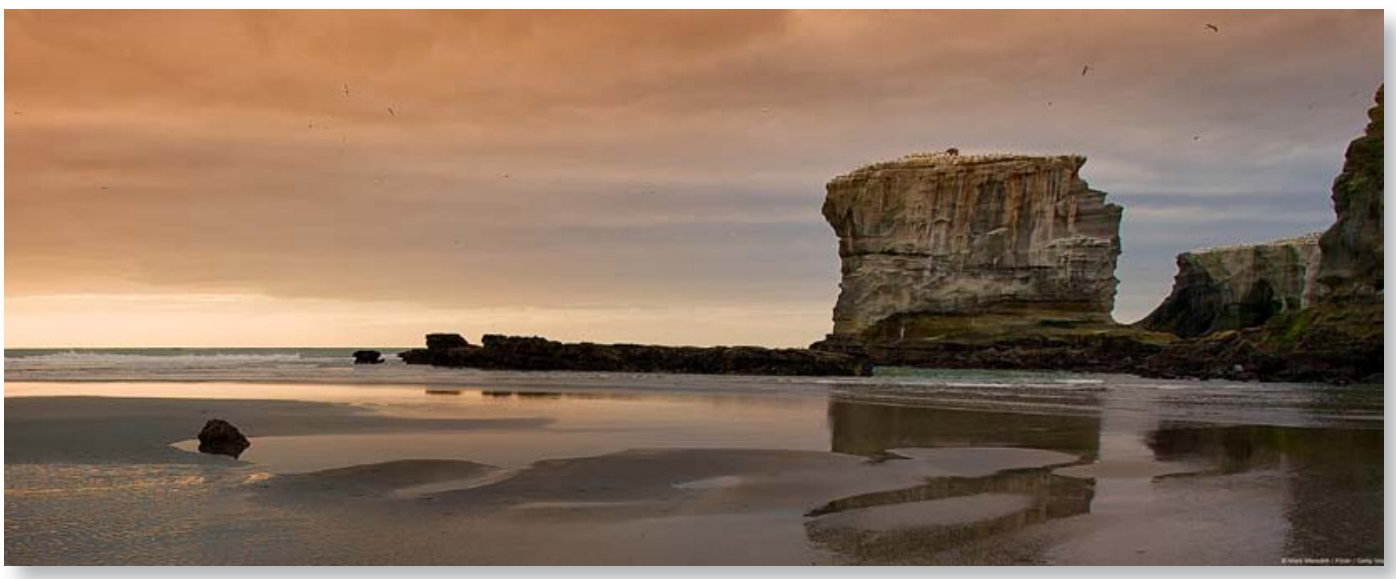

